\title{
LAS CASAS-CUARTEL DE LA GUARDIA CIVIL DURANTE LA II REPÚBLICA Y EL FRANQUISMO: LA DESCONOCIDA LABOR DE UN GRUPO DE ARQUITECTOS
}

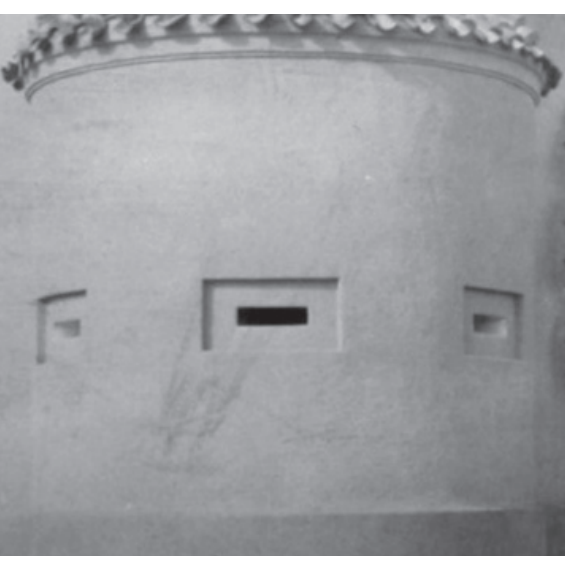

\author{
Daniel Pinzón Ayala
}

The barracks of Civil Guard during the Second Spanish Republic and the Franco regime: the unknown work of a group of architects

Boletín Académico. Revista de investigación y arquitectura contemporánea

Escuela Técnica Superior de Arquitectura. Universidade da Coruña

elSSN 2173-6723

unw.boletinacademico.com

Número 4 (2014)

Páginas 71-82

Fecha de recepción 31.10.2013

Fecha de aceptación 04.02.2014

\section{Resumen}

Este artículo pretende sacar a la luz los nombres del primer grupo de arquitectos nombrados por la Guardia Civil que, desde la República y durante el Franquismo, procedieron a la consolidación tipológica de la casa-cuartel. Esta iniciativa estatal se basó en unos modelos tipo que, con el paso de los años, fueron personalizados por cada arquitecto, generando un interesante abanico de soluciones. La trascendencia de este colectivo así como de su producción ha sido nula, a pesar de constituir uno de los impulsos constructivos más significativos en la historia del Cuerpo.

\section{Abstract}

This article aims to bring to light the names of the first group of architects of the Civil Guard that, since the Spanish Republic and during the Franco regime, consolidated the typological conformation of the barracks. This state initiative was based in several architectural models that, over the years, were customized by each architect, producing an interesting range of solutions. There is a lack of historical importance around this group and its production, although they represent one of the greatest structural impulses in the history of the Corps.

\section{Palabras clave}

Casa-cuartel, Guardia Civil, arquitectura franquista, arquitectos españoles, arquitectura contemporánea

\section{Keywords}

Barracks, Civil Guard, Franco Regime Architecture, Spanish Architects, Contemporary Architecture 

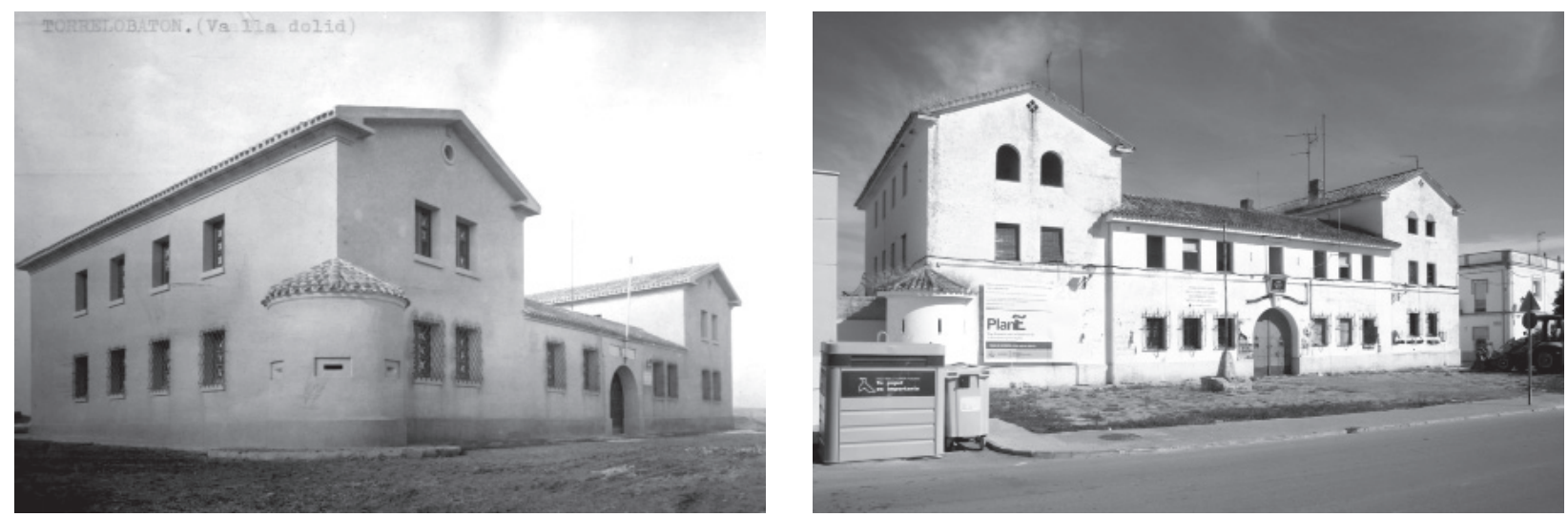

01 Ángel de la Morena Suárez, antigua casa-cuartel de Torrelobatón (Valladolid), h. 1949.

02 Enrique García-Ormaechea Casanovas, antigua casa-cuartel de Villamartín (Cádiz), 1955.

\section{INTRODUCCIÓN}

La casa es el acuartelamiento característico de la Guardia Civil. Ya en su decreto fundacional, de 13 de mayo de 1844 , se recoge la necesidad de generar un cuartel donde alojar a los guardias civiles con sus familias. Esta solución significó una apuesta muy innovadora en el contexto militar español, pues era la primera vez que una institución armada fundamentaba su labor en la convivencia conjunta de militares y civiles.

La falta de un plan de acuartelamiento, por carencias presupuestarias, hizo que la obtención de los cuarteles se acabara convirtiendo en un problema crónico para la Guardia Civil. Esto obligó a depender - fundamentalmente durante el primer siglo de existencia - de edificios en alquiler, en su mayoría caracterizados por su precariedad. Cuestiones como la habitabilidad o la correcta distribución territorial se vieron afectadas, ante las escasas posibilidades de obtener mejores inmuebles.

No fue hasta la década de los años veinte del siglo pasado cuando algunos ayuntamientos comenzaron a promover la construcción de casas-cuartel. Estos encargos recayeron normalmente en los arquitectos municipales. Ante la ausencia de referentes, se siguieron dos líneas compositivas características:

Militar; se apostó por una imagen cargada de simbolismos castrenses basada en la presencia de torreones, garitas o pretiles almenados, y una organización en planta en torno a patios, emulando fortificaciones y castillos a pequeña escala.

Civil; otros arquitectos se limitaron a construir un edificio que reuniera unas correctas condiciones de habitabilidad, acordes con las normativas en vigor,

03 Lista de arquitectos afectos a la Guardia Civil (1935/45

\begin{tabular}{|c|c|c|c|c|}
\hline \multirow[b]{2}{*}{ Arquitecto } & \multicolumn{2}{|c|}{ Orden de 6 de fulito de 1935} & \multicolumn{2}{|c|}{$\begin{array}{l}\text { Orden General del Cuerpo no. } 18 \\
21 \text { de junio de } 1945\end{array}$} \\
\hline & zona & Ccalandancias & Sector & Cowandancias \\
\hline $\begin{array}{l}\text { Pedro Sanchez } \\
\text { Sepuliveda }\end{array}$ & $1^{*}$ & 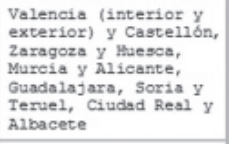 & $3^{\circ}$ Norte & $\begin{array}{l}\text { Palencia, Santander, } \\
\text { Burgos, Logrofio, } \\
\text { Guipuzcos, Vizcaya, } \\
\text { Alava, Navarra, } \\
\text { Zaragoza y Huegca }\end{array}$ \\
\hline $\begin{array}{l}\text { Enrique } \\
\text { Garcia- } \\
\text { Omaechea } \\
\text { Casanovas }\end{array}$ & $2^{2}$ & $\begin{array}{l}\text { Granada y Almeria, } \\
\text { Málaga y Cádiz, } \\
\text { Sevilia (interior y } \\
\text { exterioor) y Huelva, } \\
\text { Córdoba y Jaén }\end{array}$ & $6^{\circ} \mathrm{sur}$ & $\begin{array}{l}\text { Huelva, Cadiz, Sevilila, } \\
\text { Málaga, Granada, Jaén, } \\
\text { Córdoba y Badajoz }\end{array}$ \\
\hline $\begin{array}{l}\text { Angel de la } \\
\text { Morena Suárez }\end{array}$ & $3^{2}$ & $\begin{array}{l}\text { La Corufia, Lugo, } \\
\text { orense y Pontevedra, } \\
\text { Valladolid y Y Segovia, } \\
\text { oviedo y Leô, Burgos, } \\
\text { Palencia y Logrofio, } \\
\text { Guipúzcoa, Jilava y } \\
\text { Navarra, Santander y } \\
\text { Vizeaya }\end{array}$ & $2^{\circ}$ Oeste & $\begin{array}{l}\text { La Coruña, Lugo, } \\
\text { orenge, Pontevedra, } \\
\text { Asturias, Ieón, } \\
\text { Valladolid Y Zamora }\end{array}$ \\
\hline $\begin{array}{l}\text { José Maria de } \\
\text { la Vega Samper }\end{array}$ & $4^{*}$ & $\begin{array}{l}\text { Madrid y Avila, Toledo } \\
\text { y Cuence, Móvil de } \\
\text { Madrid, Badajoz y } \\
\text { Cáceres, } 1^{2} 2^{2} \text { de } \\
\text { Madrid, Salamance y } \\
\text { Zamora }\end{array}$ & $\begin{array}{c}9^{\circ} \\
\text { Guzman } \\
\text { e1 Bueno }\end{array}$ & $\begin{array}{l}\text { Dirección General de } 1 \\
\text { Cuerpo y Centro de } \\
\text { Instruceion }\end{array}$ \\
\hline $\begin{array}{l}\text { Javier Barroso } \\
\text { Sanchez-Guerra }\end{array}$ & $5^{2}$ & $\begin{array}{l}\text { Barcelona, Tarragona, } \\
\text { Iérida y Gerona, } \\
\text { ademas de las } 1^{*} 2^{\circ} \\
\text { de Barcelona }\end{array}$ & $4^{\circ}$ Este & $\begin{array}{l}\text { Barcelona, Tarragana, } \\
\text { Lerida, Gerona, } \\
\text { Castellón, Valencia y } \\
\text { Teruel }\end{array}$ \\
\hline $\begin{array}{l}\text { Eduardo } \\
\text { Baselga Neyra }\end{array}$ & - & - & $1^{\circ}$ & $\begin{array}{l}\text { Guadalajara, hvila, } \\
\text { Segovia, Saiamanca, } \\
\text { Cloeres, Soria y Madrid }\end{array}$ \\
\hline $\begin{array}{l}\text { Manuel Sainz } \\
\text { de V1cuña } \\
\text { Garcia-Prieto }\end{array}$ & - & - & $\begin{array}{c}5^{\circ} \\
\text { Sureate }\end{array}$ & $\begin{array}{l}\text { Almeris, Mureia, } \\
\text { Albacete, C1udad Neal, } \\
\text { Toledo, Cuenca Y } \\
\text { Alicante }\end{array}$ \\
\hline $\begin{array}{l}\text { Eduardo } \\
\text { Laforet y } \\
\text { Altolaguirre }\end{array}$ & - & - & $\begin{array}{l}7^{\circ} \text { Gran } \\
\text { Canaria }\end{array}$ & $\begin{array}{l}\text { Gran Canaria, Lanzarote } \\
\text { Y Fuerteventura }\end{array}$ \\
\hline $\begin{array}{l}\text { Tamás Machado } \\
\text { Y Méndez } \\
\text { Fernández de } \\
\text { Lugo }\end{array}$ & - & - & $\begin{array}{c}8^{\circ} \\
\text { Tenerife }\end{array}$ & $\begin{array}{l}\text { Tenerife, La Palma, } \\
\text { Gomera y Hierro }\end{array}$ \\
\hline
\end{tabular}



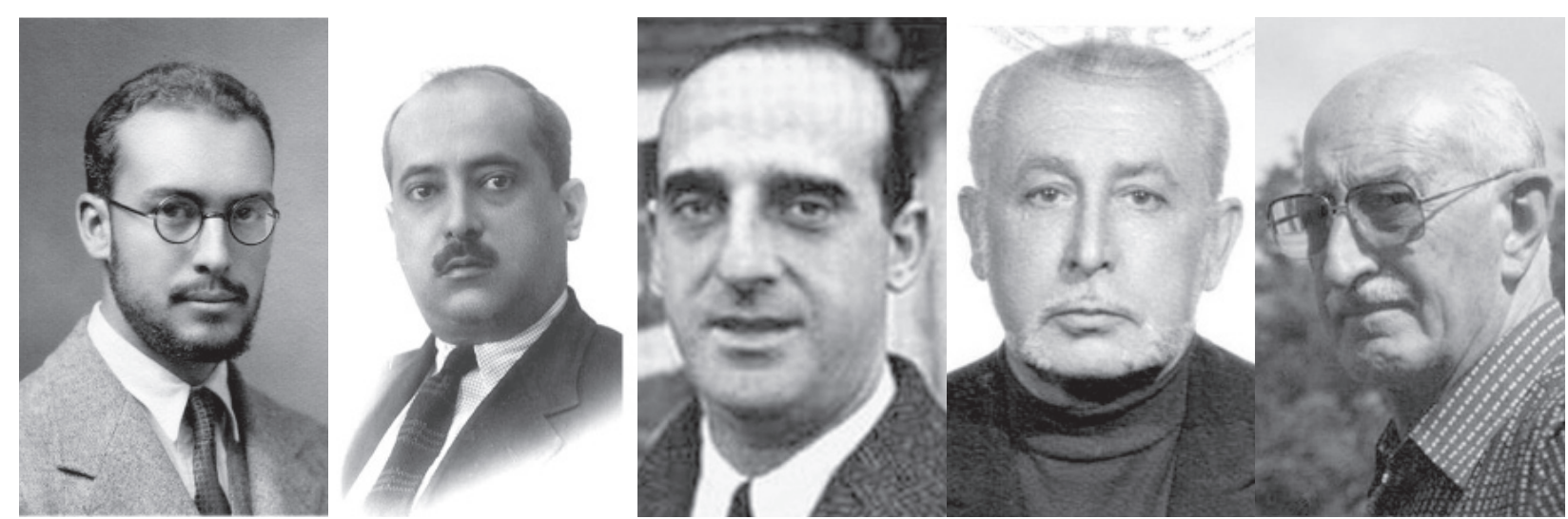

04 Enrique García-Ormaechea Casanovas; Pedro Sánchez Sepúlveda; Javier Barroso Sánchez-Guerra; Eduardo Baselga Neyra; Manuel Sainz de Vicuña García-Prieto.

con una destacada ausencia de ornamentos alusivos al carácter militar del Cuerpo.

De manera paralela, el Ministerio de la Gobernación efectuaría algunas construcciones, como ocurrió en Madrid con los acuartelamientos situados en Batalla del Salado y Guzmán el Bueno, o en Oviedo y Santander. En varios de estos proyectos destacó la figura del arquitecto $-\mathrm{y}$, finalmente, teniente coronel de la Guardia Civil- Ricardo Macarrón Piudo ${ }^{1}$.

Con la llegada de la II República se producirán los primeros movimientos desde el Estado para solucionar el problema del acuartelamiento de manera global. En este momento se pusieron los cimientos de las políticas arquitectónicas que se desarrollarían durante las décadas siguientes, de manera que un arquitecto se hace responsable de una región y procura efectuar "un modelo que se adapte a las condiciones de clima, de materiales y demás elementos que varíen según las diferentes Zonas del territorio nacional»².

Un caso particular se dio en Cataluña, en donde la Generalitat —organismo que asumió durante algunos meses las competencias sobre la Guardia Civilprocedió al encargo de una casa-cuartel estandarizable y representativa del poder regional ${ }^{3}$.

La corta duración de este periodo histórico junto con la complejidad del problema no permitió que cuajara ninguna iniciativa. El franquismo heredará y continuará esta labor hasta obtener una red estatal de casas-cuartel.

\section{LOS MODELOS DE CASAS-CUARTEL}

En la dictadura perdieron protagonismo las dos vías iniciales de obtención de acuartelamientos. Por un lado, se rescindió un número importante de contratos de arrendamiento, y por otro, los ayuntamientos dejaron de asumir la inversión que suponía ejecutar casas-cuartel de nueva planta. Este novedoso escenario permitió aspirar a una cierta homogeneización formal, imponiendo una normativa común para las viviendas y las dependencias oficiales ${ }^{4}$.

Para la implantación de esta red de acuartelamientos se decidió generar una serie de modelos, con el fin de abaratar los costes y permitir una construcción extensiva y rápida. La distribución geográfica ya establecida en el periodo anterior y la asignación de un arquitecto a cada región facilitó la creación de unos modelos comunes. Con el paso de los años, cada arquitecto desarrolló sus propias soluciones, diferenciando cada región de manera notable.

\section{LOS MODELOS NACIONALES}

En un primer momento imperaron una serie de proyectos que se aplicaron por toda la geografía nacional, independientemente de los arquitectos y sectores establecidos (Fig. 01-02). Las diferencias entre ellos se redujeron al empleo de materiales regionales o al tipo de ornamentos. Estas casas-cuartel se adscribirían a la línea militar, ya presente en los acuartelamientos promovidos por los ayuntamientos y normalizados en el periodo republicano 5 . 


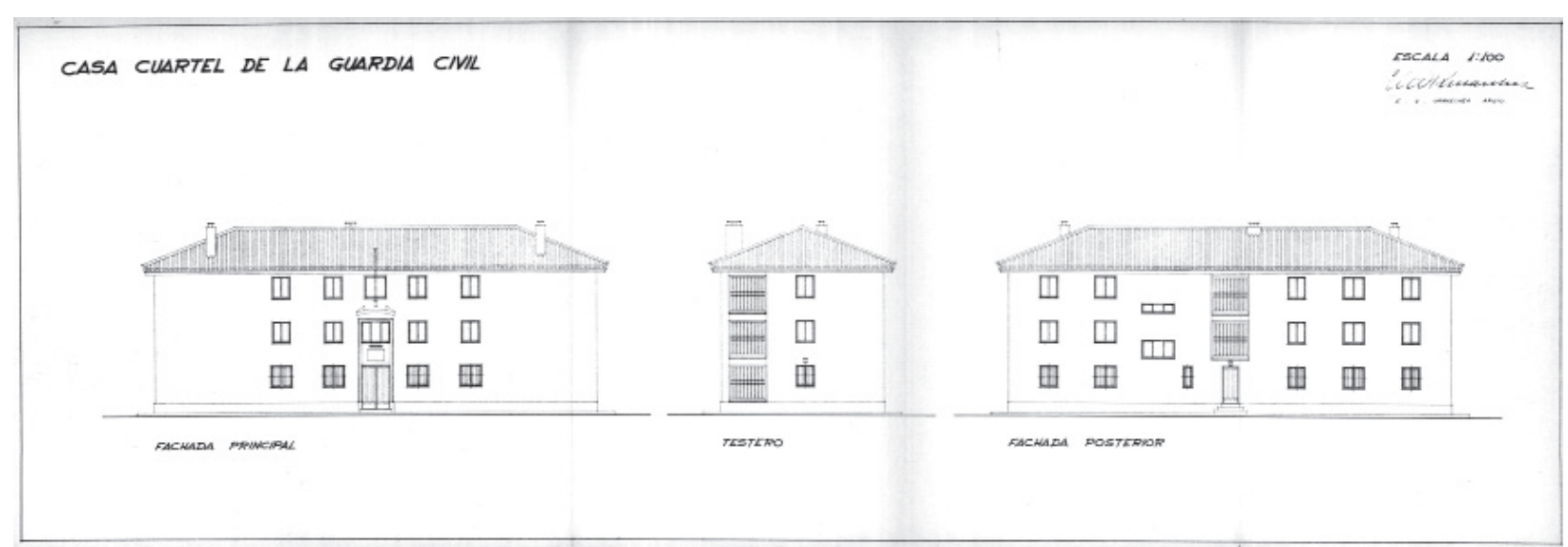

05 Enrique García-Ormaechea Casanovas, casa-cuartel de El Rocío (Huelva), h. 1965; plano tipo de fachadas.
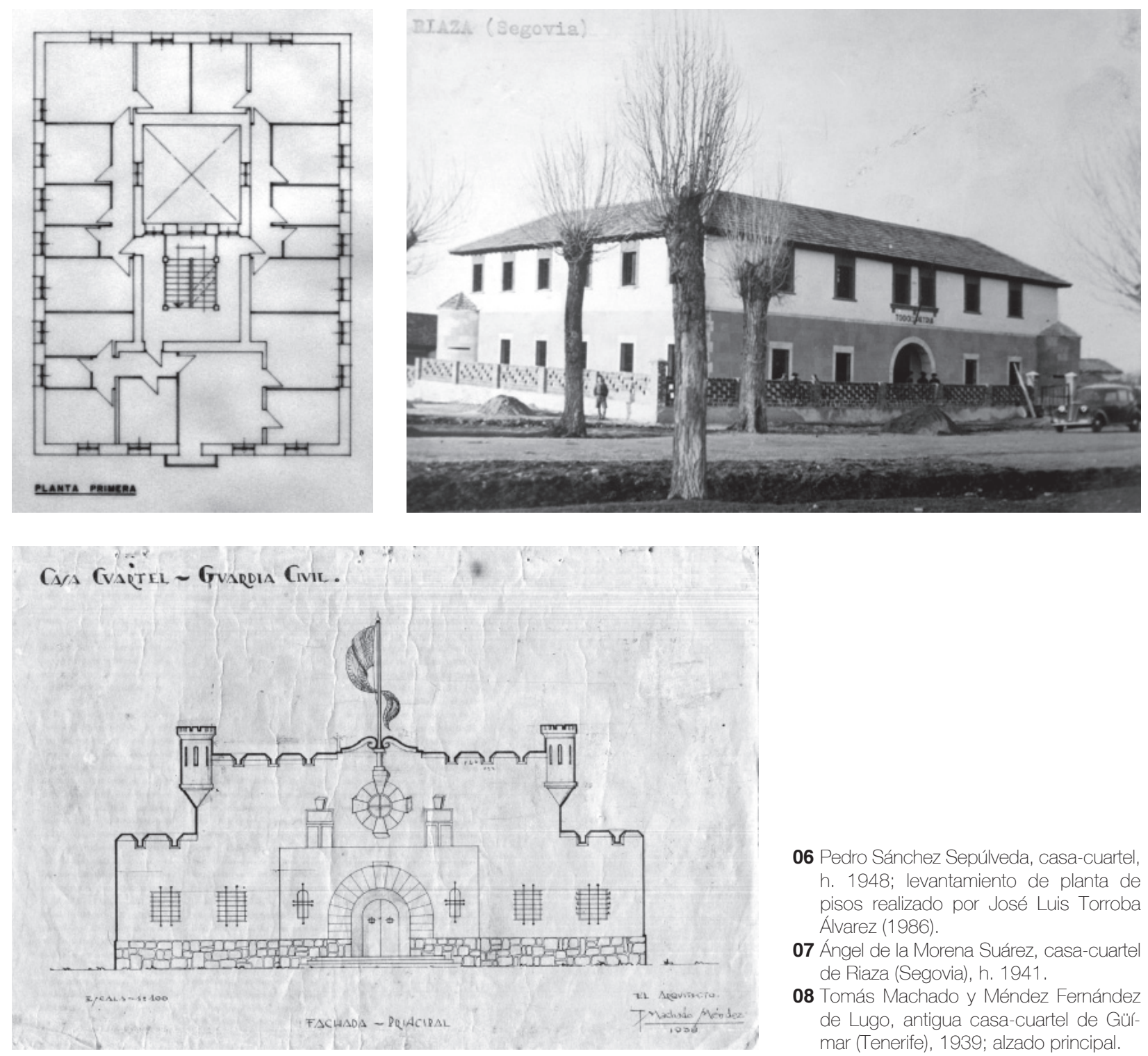

06 Pedro Sánchez Sepúlveda, casa-cuartel, h. 1948; levantamiento de planta de pisos realizado por José Luis Torroba Álvarez (1986).

07 Ángel de la Morena Suárez, casa-cuartel de Riaza (Segovia), h. 1941.

08 Tomás Machado y Méndez Fernández de Lugo, antigua casa-cuartel de Gürmar (Tenerife), 1939; alzado principal. 


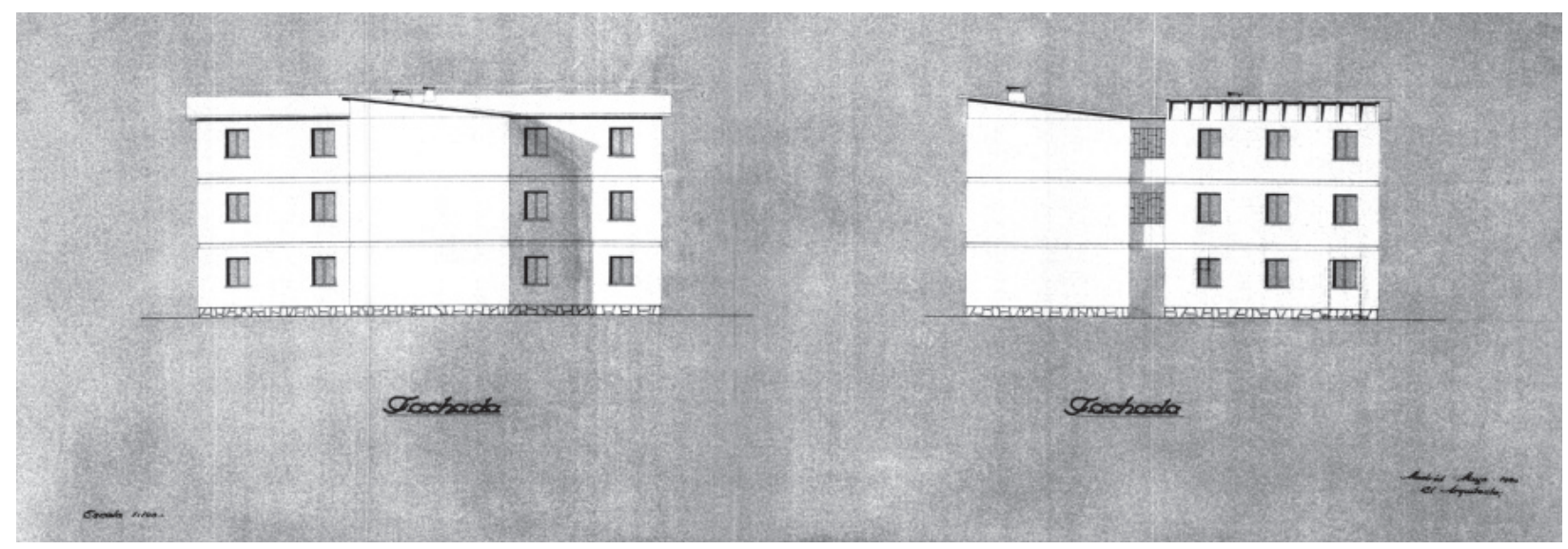

09 Eduardo Baselga Neyra, casa-cuartel de Zarza de Granadilla (Cáceres), 1960; plano tipo de fachadas.

Se ajustaban, básicamente, a una organización en planta en forma de U, yuxtaponiendo las dependencias oficiales y las viviendas, con un único acceso y unos espacios interiores muy definidos. A partir de un programa básico se formalizaron diferentes soluciones, alterando los volúmenes de fachada o abriendo el patio principal a ampliaciones, según la categoría del acuartelamiento.

En casos como la Dirección General de Regiones Devastadas (DGRD) o el Instituto Nacional de la Colonización, se preferirían edificios cerrados en torno a un patio central. Tal y como describe Gabriel Ureña en una de las escasas referencias bibliográficas en torno a este tipo de edificios ${ }^{6}$, las casas-cuartel elegidas en la etapa franquista - sobre todo en el periodo autárquico - respondieron a una imagen de casticismos regionalistas, con diferentes expresiones, debiendo cumplir con una serie de objetivos. El edificio, polifamiliar, burocrático y dotado de cuadras para caballerías, tenía que alternar una triple funcionalidad: mostrar un carácter defensivo y cerrado de su armazón exterior; un casticismo regionalista en patios y fachadas; y alternar espacio exterior-cerrado/espacio interior-abierto en los patios ${ }^{7}$.

La apuesta por una estética defensiva consiguió transmitir a la sociedad de entonces la idea de seguridad pública de las políticas franquistas. Estos modelos, aunque característicos de las dos primeras décadas de dictadura, fueron constantes en todo el periodo, tendiendo con el paso del tiempo hacia la simplificación y la eliminación de sus ornamentos.

\section{LOS MODELOS SECTORIALES}

Fruto de la labor particular de cada arquitecto surgieron nuevas soluciones de casas-cuartel. El denominador común de estos proyectos se basaba en los programas, que permanecieron sin cambios importantes, pero se diferenciaban formalmente de manera notable. Se caracterizaron por implementar construcciones muy apartadas de los modelos nacionales, en una clara línea de modernización y mayor racionalización de estos complejos, mostrando ciertas semejanzas con los ejemplos habitacionales de las periferias urbanas.

La Orden de 20 de abril de 1956, emitida conjuntamente por los ministerios de la Gobernación y Trabajo, establecía en su artículo tercero la obligación de aprobar unos proyectos tipo previamente formalizados por los servicios técnicos de la Guardia Civil. Se consolidaba así una determinada línea de trabajo, pero no impedía que cada arquitecto aportara sus propias alternativas y las estandarizara.

Las nuevas soluciones superaron la estética militar anterior. En todas ellas se favoreció la separación espacial y volumétrica de los dos usos principales, aunque todavía varios arquitectos mantendrían ordenaciones de conjunto ciertamente compactas o en donde al menos un edificio seguía aglutinando oficinas y pabellones.

De manera general se evidenció una pérdida sistemática de la idea del patio central como elemento perfectamente delimitado. Como consecuencia de presupuestos más austeros se produciría una disminución de 

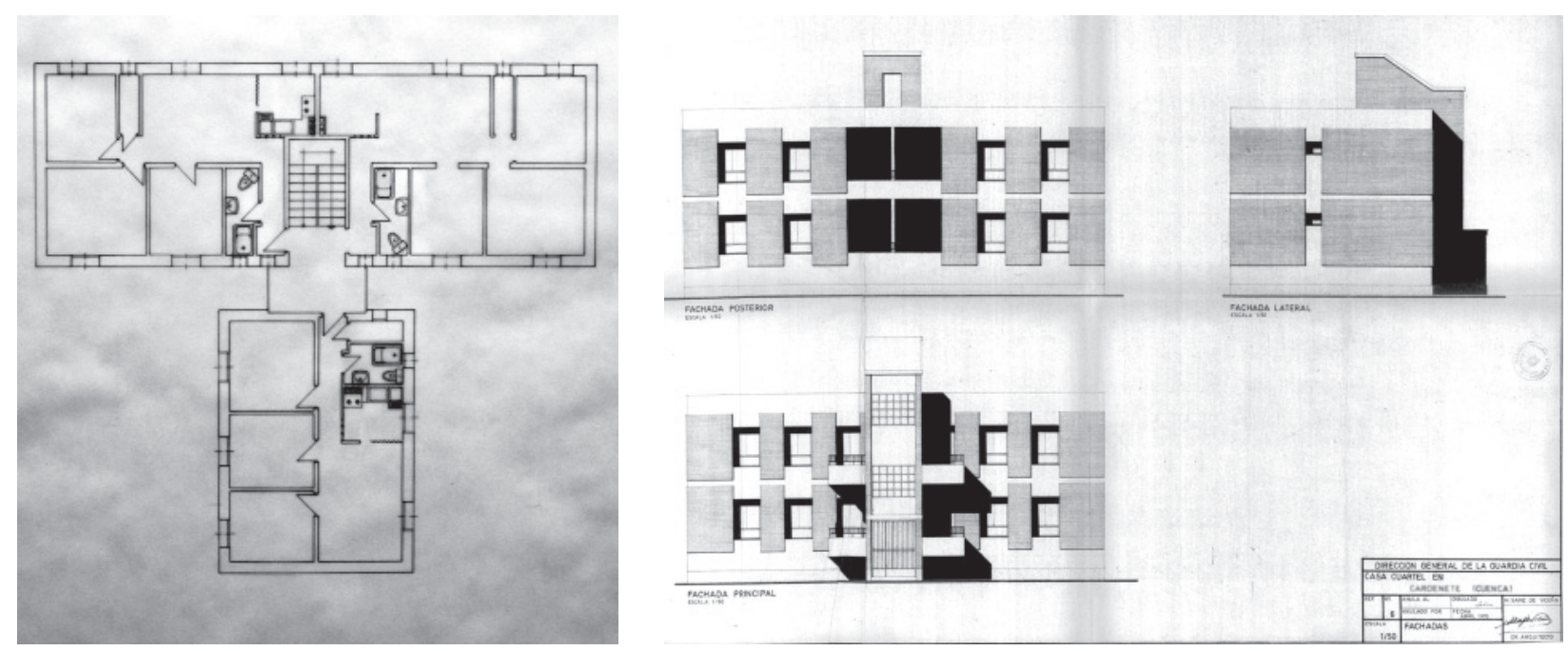

10 Eduardo Baselga Neyra, casa-cuartel, h. 1964; levantamiento de planta de pisos realizado por José Luis Torroba Álvarez (1990). 11 Manuel Sainz de Vicuña García-Prieto, casa-cuartel de Cardenete (Cuenca), 1970; plano tipo de fachadas.

los metros cuadrados de ocupación y una progresiva ausencia de ornamentos militares, recuperando de este modo aquella otra línea civil de los años veinte.

\section{LOS ARQUITECTOS}

Por la Orden de 3 de julio de 1931, se aprobó el primer concurso por el que se procedía al nombramiento de los arquitectos que quedarían afectos a las diferentes direcciones generales del Ministerio de la Gobernación. Dos de ellos recalaron en el servicio de la Guardia Civil. La Orden de 5 de octubre resolvió que éstos serían José María de la Vega y Samper y Enrique García-Ormaechea Casanovas.

Aunque fueron diferentes las reformas que se efectuaron en este periodo - como la desvinculación de la Guardia Civil del Ministerio de la Guerra-, hay que destacar la que se lleva a cabo un año antes del comienzo de la guerra civil. Por aplicación del cuarto artículo del Decreto de 13 de junio de 1935, se convocó el concurso para cubrir tres plazas de arquitectos para la Guardia Civil, con idea de equiparar la organización militar con la técnica, de manera que se pudiera asignar a un técnico por Zona ${ }^{8}$. De aquí resultó un primer quinteto de arquitectos, como recogió la Orden de 6 de julio de 1935 (Fig. 03).

Pasada la guerra civil, la Dirección General de la Guardia Civil mantuvo a los cinco arquitectos que, por concurso, fueron nombrados en el periodo republicano, sumándoles cuatro más por designación directa, tal y como se procedería a partir de entonces. De este modo, mediante la Orden General del Cuerpo número 18 , de 21 de junio de 1945 , se conforma un grupo de nueve técnicos a los que se les asignará un Sector, organización territorial que se independizaría de la establecida para la administración y los servicios. La idea era equilibrar cada uno con un número similar de provincias, salvo en el caso canario, en donde se nombraron dos arquitectos (Fig. 03). Este colectivo y su reparto territorial se mantuvieron durante varios años, con sustituciones esporádicas, hasta que a principios de los años setenta se produjeron nuevas incorporaciones y modificaciones en los límites sectoriales.

\section{BREVES RESEÑAS BIOGRÁFICAS ${ }^{9}$}

Básicamente podemos centrar nuestra atención en once arquitectos, los nueve descritos más dos sustituciones que destacaron por su gran aportación (Fig. 04). Este grupo de técnicos se puede considerar como el primero que permaneció afecto a la Guardia Civil con cierta continuidad, y permitió dar estabilidad a unas políticas de implantación de casas-cuartel muy ambiciosas. Todavía en la actualidad estos acuartelamientos suponen un número importante de los que se encuentran en uso.

José María de la Vega Samper (1900/80, t. 1926) fue el técnico que estableció la relación más peculiar con la Guardia Civil, ya que tras estar asignado a diferentes provincias del centro y oeste del país, finalmente recalaría en la Dirección General del Cuerpo y en su Centro de Instrucción ${ }^{10}$. Sus intervenciones más destacadas se produjeron en grandes acuartelamientos, como 


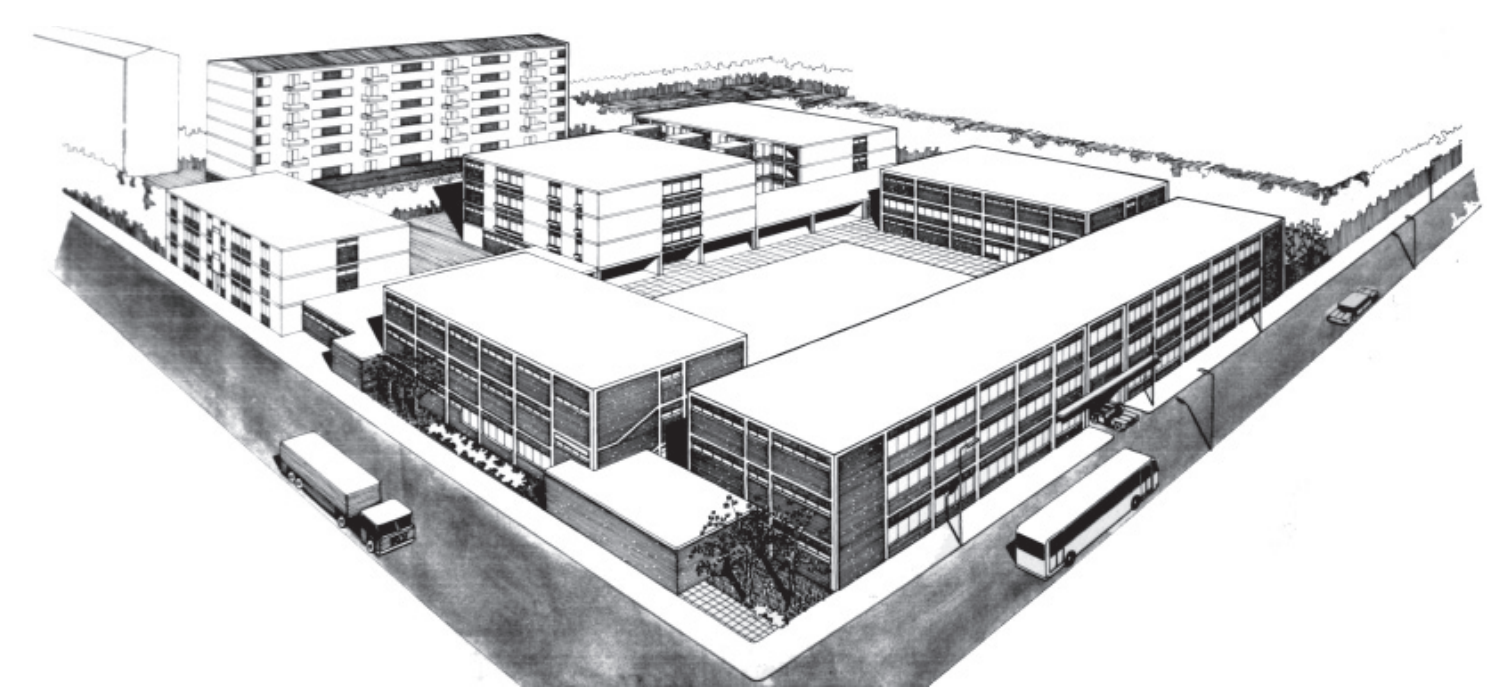

12 Manuel Sainz de Vicuña García-Prieto, Comandancia de Toledo, 1964; perspectiva del conjunto.

la adaptación para la Guardia Civil del cuartel de La Victoria en Córdoba en la etapa republicana, junto con Enrique García-Ormaechea o, tras la guerra civil, dirigiendo las diferentes reformas y ampliaciones en los cuarteles de Guzmán el Bueno y de Batalla del Salado en Madrid. Se le conoce sobre todo por su labor para la Compañía Telefónica Nacional de España ${ }^{11}$, aunque también fue arquitecto de la Dirección General de Prisiones, de la Dirección General de Asuntos Eclesiásticos, de la Junta Provincial de Protección de Menores de Madrid y de la Dirección General de Regiones Devastadas (DGRD). Algunas de sus obras han sido recogidas en diferentes investigaciones ${ }^{12}$.

Enrique García-Ormaechea Casanovas (1907/83, t. 1930) fue el arquitecto que tuvo la relación más prolongada con la Guardia Civil. El recorrido proyectual de este arquitecto ejemplifica la evolución que experimentó la casa-cuartel estatal en el pasado siglo veinte, pues proyectó en los tres últimos periodos históricos: república, dictadura y actual democracia. El hecho de permanecer ligado a Andalucía y Badajoz posibilitó que llevara a cabo una gran cantidad de proyectos. Destaca, por un lado, la transformación de los modelos nacionales a unos niveles de calidad que no alcanzaron sus compañeros, y por otro, el abanico de variantes tipológicas que mostró con soluciones propias que aportan una variedad estética muy interesante a la Zona sur (Fig. 05). Al ser funcionario del Ministerio de la Gobernación, extendió su labor a otras instituciones con colaboraciones esporádicas, como en la DGRD o en la Obra Sindical del Hogar ${ }^{13}$.
Pedro Sánchez Sepúlveda (1891/66, t. 1920) estableció una corta relación con el Instituto, desvinculándose a finales de los años cuarenta. Su producción contiene un tipo de acuartelamiento característico, a modo de gran caserón con un pequeño patio interior (Fig. 06), que difiere de las propuestas de los modelos nacionales, aunque fueron éstos los que implantó en mayor número. También fue arquitecto del Ministerio de la Educación ${ }^{14}$. En 1934 fue nombrado vocal del Consejo Nacional de Cultura, ejerciendo en plena guerra civil como arquitecto del Servicio de Defensa del Patrimonio Artístico Nacional y, tras la contienda, también para la DGRD y como arquitecto de Construcciones Civiles. Además, destacó en su labor empresarial, adquiriendo y dirigiendo la fábrica de cerámica La Covadonga, en Alcázar de San Juan.

Javier Barroso Sánchez-Guerra (1903/90, t. 1927) estableció una larga relación con el Cuerpo, continuada por su hijo Juan José Barroso Ladrón de Guevara. Se mantendría fiel a la implantación de los modelos nacionales, produciendo además soluciones propias que no llegaron a un número significativo de ejemplos. Colaboró con diferentes instituciones tras la guerra civil en distintos procesos de rehabilitación: DGRD, Ministerio de Justicia, Ciudad Universitaria, etc., además de establecer colaboraciones esporádicas con otros compañeros ${ }^{15}$. En ciertos ámbitos, su relación con el mundo del fútbol -en los planos deportivo, de gestión y técnico- es la que más ha trascendido.

Ángel de la Morena Suárez (1904/91, t. 1932) desarrolló fundamentalmente su labor en la etapa 


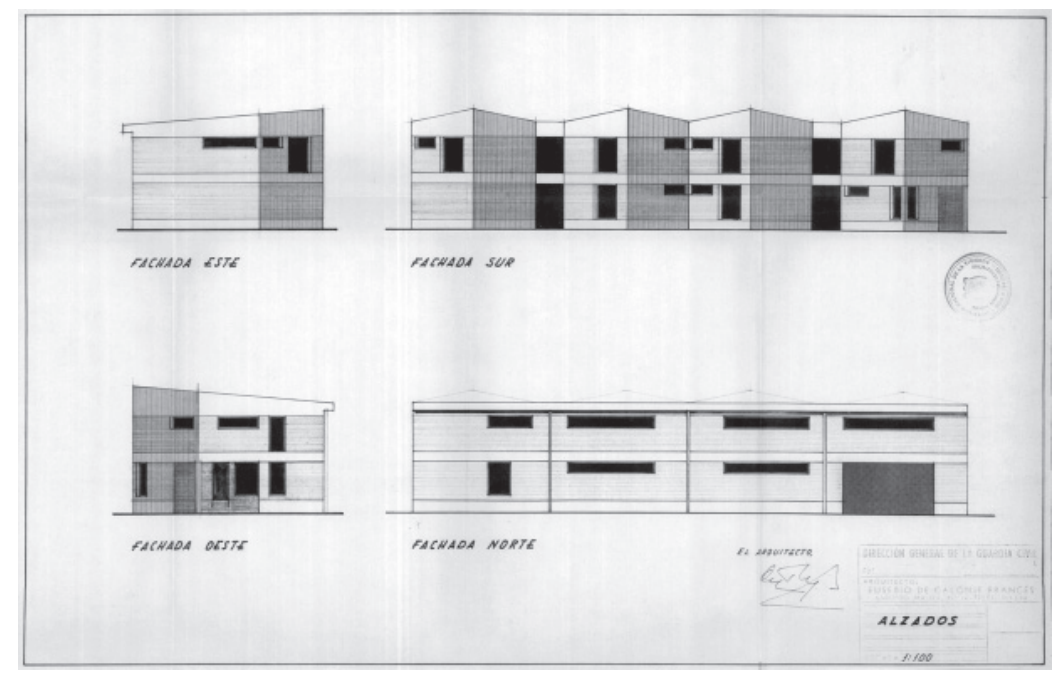

13 Eusebio de Calonje Francés, casa-cuartel de Cornago (La Rioja), h. 1970; plano tipo de fachadas.

denominada de los modelos nacionales, aunque se conoce algún ejemplo propio basado en los criterios de los tipos entonces establecidos (Fig. 07). Al igual que Sánchez Sepúlveda, simultaneó su labor con la de arquitecto escolar, desapareciendo su relación con la Guardia Civil en 1956.

Eduardo Laforet y Altolaguirre (1891/54, t. 1922) fue junto con Sánchez Sepúlveda, el más veterano de este grupo de arquitectos, aunque también el más desconocido en cuanto a su labor para la Guardia Civil, ya que no se le conoce proyecto alguno. Esto se puede deber a la limitada producción de acuartelamientos en la provincia de Las Palmas de Gran Canaria, y a las actuaciones de otros arquitectos peninsulares en dichas islas, tales como Ángel de la Morena, Felipe GarcíaEscudero o Javier de Zuazo Bengoa ${ }^{16}$. Fue arquitecto municipal y del Cabildo de Las Palmas, además de profesor y director de la actual Escuela Politécnica $(1945 / 53)$. Son varias las obras suyas recogidas en diferentes trabajos de investigación, así como su aportación a la arquitectura cinematográfica de Las Palmas en el primer tercio del siglo $\mathrm{XX}^{17}$.

Tomás Machado y Méndez Fernández de Lugo (1908-2003, t. 1939) estableció su relación con la Guardia Civil antes de su nombramiento, localizándose proyectos para ayuntamientos tinerfeńos desde 1939. Es característico su extendido modelo de casa-cuartel que difiere de todos los desarrollados en el país, similar a una fortificación, cargado de un número importante de elementos militares y de referencias autóctonas (Fig. 08). Es el único que posee una tesis monográfica sobre su vida y obra, ya que fue unos de los principales referentes de la arquitectura canaria del siglo $\mathrm{XX}^{18}$.

Eduardo Baselga Neyra (1916/93, t. 1944) sumaría a sus casas-cuartel las viviendas que proyectara para otros organismos afines a la Guardia Civil, como el Patronato de Viviendas o la Asociación Mutua Benéfica, en algunos casos en colaboración con su hijo José Ignacio Baselga García-Escudero. Su proyecto más característico de casa-cuartel fue un modelo en $\mathrm{T}$ con cubierta inclinada a un agua, el cual adaptaría a diferentes programas de acuartelamiento (Fig. 09-10). De su producción arquitectónica ha trascendido muy poco, salvo las viviendas de la calle Virgen de Luján 20 en Sevilla (1959/63), reflejadas en el PGOU, o el segundo premio obtenido junto con Joaquín Vaquero Palacios en el concurso del estadio de fútbol del Real Madrid (1944) ${ }^{19}$.

Manuel Sainz de Vicuńa y García-Prieto (1916, t. 1944) es uno de los arquitectos más laureados de este grupo, condecorado con la $3^{a}$ Medalla en la Exposición Nacional de Bellas Artes (1948) y la Encomienda de Número del Mérito Civil, a propuesta de la Guardia Civil. Realizó unos doscientos proyectos de casascuartel, desarrollando diferentes variantes de los modelos nacionales. Formalizaría un modelo tipo de bloque para viviendas y otro para dependencias oficiales, muy presentes en su Sector (Fig. 11), aunque no renunciaría en ciertos casos, preferentemente en las comandancias, a una imagen más contemporánea (Fig. 12). Su obra más renombrada es, sin lugar a dudas, la fábrica de la Perfumería Gal en Alcalá de Henares (1956/63), lo 


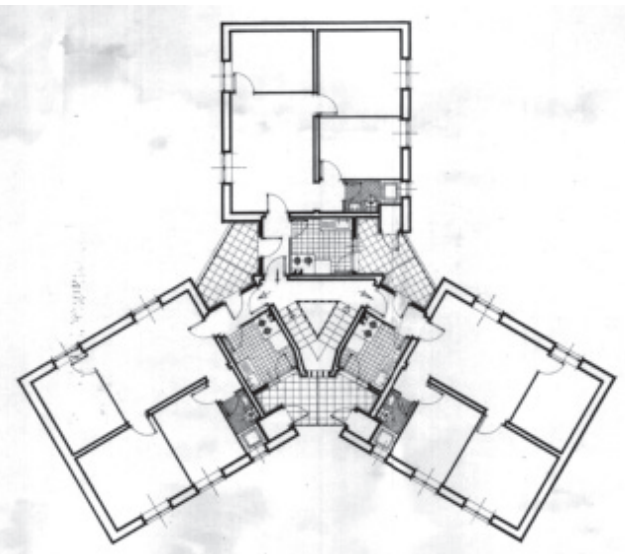

STlarba de pisos

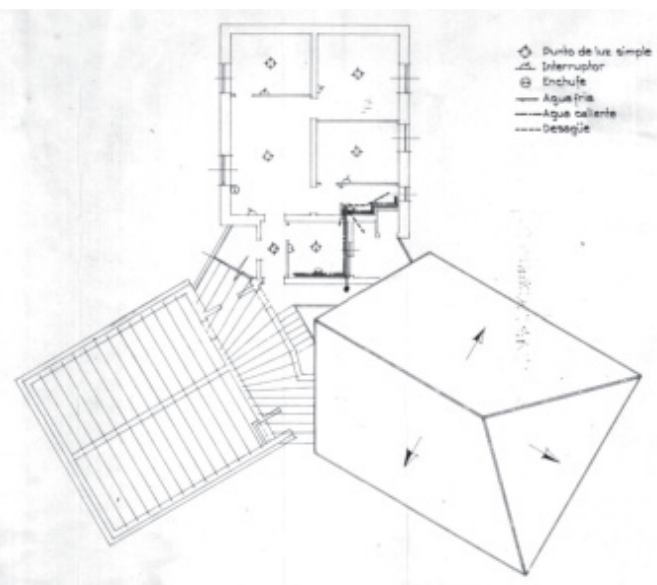

Tleurta de fajidido y cubielbas

14 Felipe García-Escudero Torroba, antigua casa-cuartel de Llanes (Asturias), h. 1962; plano tipo de planta de pisos y planta de forjado y cubiertas.

15 Felipe García-Escudero Torroba, casa-cuartel de Celanova (Ourense), 1970; plano tipo de fachadas y sección.

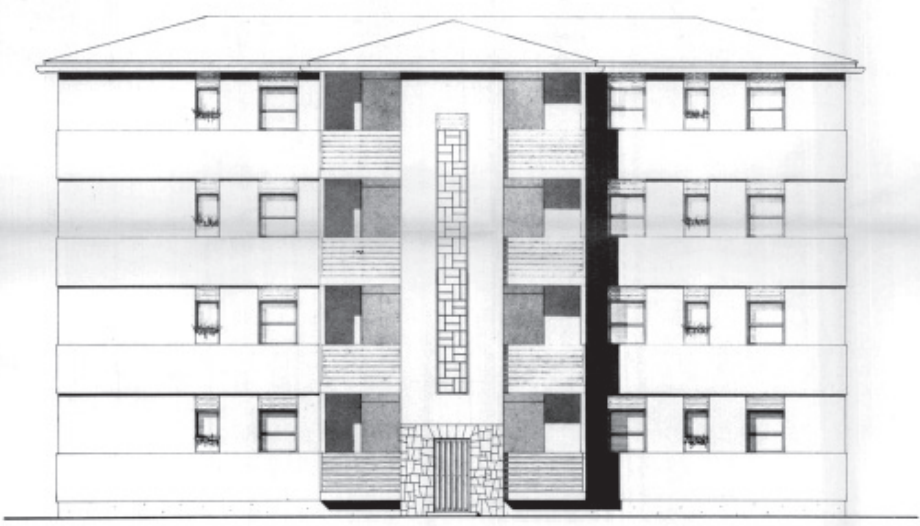

FACHADA POSTERIOR

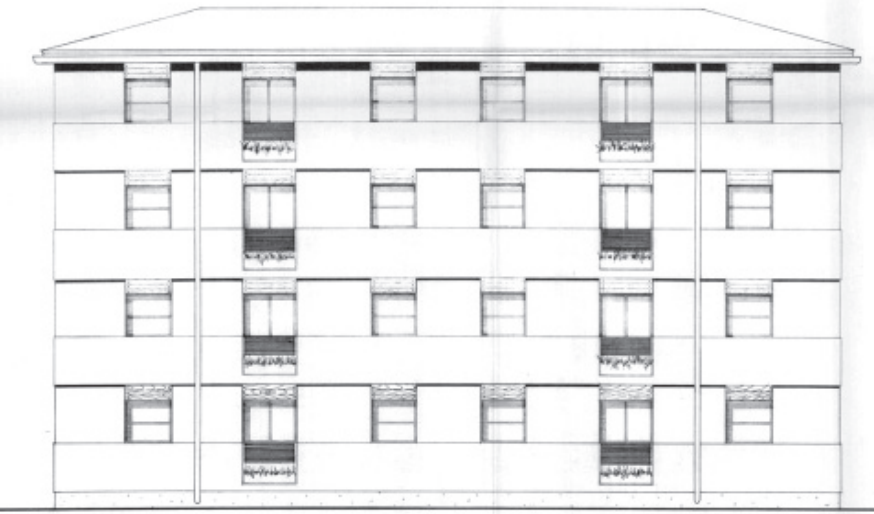

FACHADA PRINCPAL

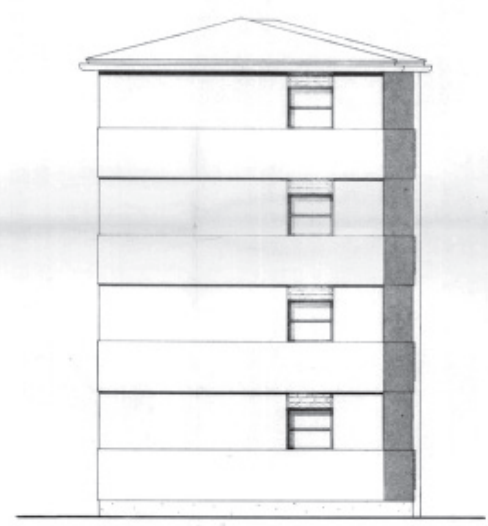

FACHAOA LATERAL

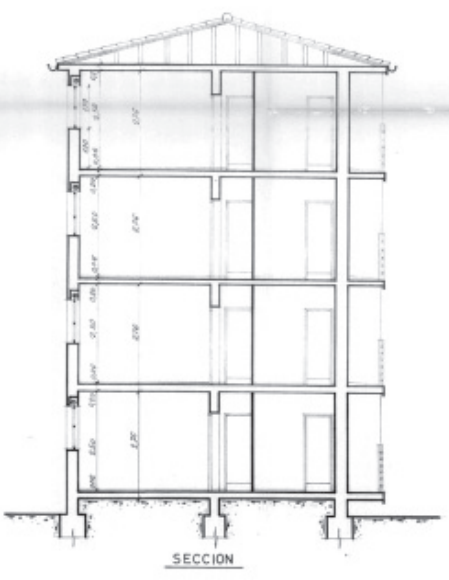

(2) 
que le ha otorgado un gran reconocimiento dentro del ámbito arquitectónico industrial ${ }^{20}$. Además desarrolló diferentes cargos públicos, tanto al frente de la Dirección General de Turismo - realizando un importante número de Paradores Nacionales - como en la Dirección General de Establecimientos Penitenciarios ${ }^{21}$. Otras obras suyas han sido recogidas en diferentes catalogaciones, principalmente las realizadas en colectivo ${ }^{22}$.

Eusebio de Calonje Francés (1917/79, t. 1943) reemplazó a Pedro Sánchez Sepúlveda en el 3o Sector, manteniendo su relación con la Guardia Civil hasta su fallecimiento. Al igual que sus compañeros, comenzó empleando los modelos nacionales, para acabar aportando acuartelamientos propios, entre los que sobresalen las piezas longitudinales con fachada trasera en zigzag (Fig. 13), de gran similitud a los que implantara, junto con Rafael Aburto, en la parcela D en el Gran San Blas, Madrid (1959/62) 23. Su obra más destacada es la iglesia de Nuestra Señora de los Ángeles, en Miranda de Ebro $(1951 / 58)^{24}$.

Felipe García-Escudero y Torroba (1919/95, t. 1948) prosiguió la labor de Ángel de la Morena en el 2o Sector, desarrollando una profusa labor para la Guardia Civil. Incorporaría un importante número de soluciones propias, entre las que destacan los modelos de estrella de tres puntas (Fig. 14) y los bloques con fachadas de bandas horizontales bicolores (Fig. 15). Uno de sus edificios más conocidos es el que proyectó junto a Ramón Vázquez Molezún para los laboratorios de investigación de Standard Eléctrica ITT, actual edificio Anaya, Madrid (1969/73) 25 .
No podemos terminar este artículo sin mencionar a un importante número de aparejadores que, distribuidos en Subsectores - equivalentes territorialmente a una provincia-, realizaron una labor complementaria de gran importancia. En muchos casos su misión resultaba fundamental para asegurar la colaboración de los ayuntamientos y garantizar la culminación de las obras, gracias a su cercanía a las mismas.

\section{CONCLUSIONES}

El hecho constructivo de la casa-cuartel se muestra como un complejo fenómeno fruto de diferentes impulsos y en distintas etapas. A la organización territorial establecida en la República, la dictadura franquista aportó el impulso necesario para generar un parque inmobiliario que ha sido fundamental para entender la actual organización periférica de la Guardia Civil.

Gracias al grupo de arquitectos reseñados se pudo conformar el concepto tipológico de casa-cuartel y de su programa híbrido laboral-residencial, consolidándolo y estableciendo su necesaria evolución. Con la modernización de sus propuestas, alejadas de influencias militarizadas, se abriría una línea proyectual que ha proseguido hasta la actualidad.

El conocimiento de este colectivo permite aportar un plus de interés sobre las casas-cuartel, al tiempo que se rescata a muchos de ellos del olvido en el que se encontraban. Se evidencia así la necesidad de profundizar en la labor de estos arquitectos y en sus proyectos, reclamando el espacio que merecen dentro de la historia de la arquitectura espańola contemporánea. 


\section{Notas}

1. Ricardo Macarrón Piudo (1884/73, t. 1922) desarrolló en paralelo su carrera militar dentro de la Guardia Civil con la de arquitecto. Como técnico del Ministerio de la Gobernación estuvo ligado a la Dirección General de Seguridad y a la de Beneficencia General. Más tarde, tras un papel muy activo durante la guerra civil como Gobernador Civil de Pontevedra y presidente de la Comisión Depuradora, pasaría al Servicio de Recuperación del Patrimonio Artístico Nacional. Su obra más conocida es el colegio del Santo Ángel para huérfanos de la Policía Nacional, en Carabanchel, Madrid (1926/36); cf. VV.AA., Arquitectura de Madrid. Periferia (Madrid: Fundación Arquitectura COAM, 2007), 660.

2. «Decreto disponiendo que la Inspección general de la Guardia Civil tenga, desde ahora, a sus inmediatas órdenes, para el estudio y ejecución de los acuartelamientos, cinco Arquitectos, uno por cada una de las cinco Zonas en que se distribuyen los Tercios del Instituto", Gaceta de Madrid 166 (15 de junio de 1935): 2196.

3. La Consellería de Governació encargó un anteproyecto de casa-cuartel a Josep Lluis Sert, Josep Torres Clavé y Joan Baptista Subirana, para las fuerzas de caballería y de infantería en Barcelona; cf. Josep Emili Hernández-Cros, «Caserna per a Unitat d’Infanteria (CUI). Anteproyecto de cuartel para las fuerzas de Infantería de la Guardia Civil de guarnición en Barcelona», Cuadernos de Arquitectura y Urbanismo 94 (1973): 26-27.

4. El Instituto Nacional de la Vivienda acabaría aportando el soporte económico necesario para la ejecución de las casas-cuartel, imponiendo como condición el cumplimiento de sus normativas para las viviendas.

5. Boletín Oficial del Cuerpo (24 de junio de 1935): 716.

6. Cf. Daniel Pinzón Ayala, «La casa-cuartel de la Guardia Civil: la puesta en valor de una arquitectura marginal», Revista de Historia y Teoría de la Arquitectura 10-11 (2008-2009): 124-127.

7. Gabriel Ureña Portero, Arquitectura y Urbanística Civil y Militar en el Periodo de la Autarquía (1936-1945) (Madrid: Istmo, 1979$), 131$.

8. Las Zonas fueron creadas por Real Orden Circular de 20 de mayo de 1926, erigiéndose como la mayor unidad dentro de la organización periférica de la Guardia Civil. Con el Real Decreto 367/1997, de 14 de marzo, las Zonas se hicieron territorialmente coincidentes con cada comunidad autónoma.

9. Muchos de los datos biográficos de los arquitectos reseñados proceden de los familiares de los arquitectos y del Servicio Histórico del COAM, en la figura de su coordinador Alberto Sanz Hernando.

10. En 1951 el 9o Sector abarcaba la Zona del Protectorado marroquí y se encontraba asignado a Alejandro Blond González (1916, t. 1944), autor de la casa-cuartel de Ceuta.

11. Cf. Francisco Javier García Algarra, «De Gran Vía al Distrito C. El patrimonio arquitectónico de Telefónica» (Tesis Doctoral, UNED, 2001) y «Las primeras centrales telefónicas en Galicia», Boletín Académico 2 (2012): 1-8.

12. Destacan la Cárcel de Carabanchel (1939), junto con Vicente Agustí Elguero y Luis de la Peńa Hickman, y el colegio Cabrini en Madrid (1950/53). También han sido dignas de atención las viviendas proyectadas en la calle Capuchinos Viejos 1, en Valladolid (1938); cf. VV.AA., Arquitectura de Madrid. Periferia, 680 y 268; Celestino García Braña y Fernando Agrasar Quiroga (eds.), Arquitectura Moderna en Asturias, Galicia, Castilla y León. Ortodoxia, márgenes y transgresiones (A Coruña: COAAs, COAG, COACyLE, COAL: 1998), 313.

13. Para la DGRD realizó la Comandancia de la Guardia Civil de Cádiz, junto con Francisco Echenique Gómez (1954) y, en colectivo, la colonia Tercio y Terol (1940/51); cf. Archivo General de la Administración (AGA), Fondo Dirección General de Regiones Devastadas, caja 153; Luis Moya Blanco, «Los Barrios del Tercio y El Terol», en Carlos Sambricio (ed.), Un siglo de vivienda social (1903/2003), Tomo I (Madrid: Nerea, 2003), 292-293. Para la OSH dirigió la construcción del Grupo Juan Tornero (1956/61) junto con Alfonso Villamarín García y, como arquitecto jefe, el barrio III del distrito de Moratalaz (1970), ambas actuaciones en Madrid; cf. VV.AA., Arquitectura de Madrid. Periferia, 725 y 398-399.

14. Como intervenciones para el ministerio de la Educación se han catalogado las realizadas en las escuelas nacionales en El Álamo (1924/25) y las de Santa María (1934), Marcelo Usera (1939/40) y Jorge Manrique (1940/43) en Madrid; cf. Félix Cabrero Garrido, Fátima García Lledó y María Cristina García Pérez, «El Álamo», en VV.AA, Arquitectura y desarrollo urbano Comunidad de Madrid Zona sur, vol. X (Madrid: Dirección General de Arquitectura y Vivienda, Fundación COAM, Fundación Caja Madrid, 2004), 48-52; Amparo Berlinches Acín (dir.), Arquitectura de Madrid. Casco Histórico (Madrid: Fundación Arquitectura COAM, 2003), 273; VV.AA., Arquitectura de Madrid. Periferia, 553 y 552.

15. Junto con Luis Gutiérrez Soto firmó el Mercado de Salamanca (1942); en Madrid actuó en el Instituto Nacional de Previsión Espańola con Enrique García-Ormaechea y el ingeniero Carlos Fernández Casado (1946); reformó, junto con Fernando Cánovas del Castillo, el Palacio de la Equitativa (1954/55); en colaboración con su hijo Juan José Barroso proyectaría la iglesia parroquial de San Mateo (1967/70) y también actuaría en la obra colectiva del barrio de Moratalaz (1970); cf. García Braña y Agrasar Quiroga, Arquitectura Moderna, 315; Javier Barroso Sánchez-Guerra y Enrique García-Ormaechea Casanovas, «El edificio sede del Instituto Nacional de Previsión en Madrid», Revista Nacional de Arquitectura 58-59 (1946): 207-218; Berlinches Acín, Arquitectura de Madrid. Casco Histórico, 213; VV.AA., Arquitectura de Madrid. Periferia, 398-399.

16. Javier de Zuazo Bengoa (1922, t. 1950) consta en la lista de colegiados del COAM como afecto a la Guardia Civil a principio de los sesenta, con proyectos en la provincia de Las Palmas y, en algún caso, únicamente como director de obra.

17. Entre sus obras sobresalen el chalet Frondt Siemens (1924); la intervención en el conjunto urbano de Perojo; la modificación y consecución del edificio del Cabildo Insular (1941) — obra original de Miguel Martín Fernández de la Torre— o la sede del Gobierno Civil (1942); cf. www.archxx-sudoe.es, no. ficha LP00014 y LP00015; Óscar Naranjo Barrera, "Construcción de un análisis», en VV.AA., El cabildo insular y la ciudad racionalista [catálogo de la exposición] (Las Palmas: Cabildo Insular de Gran Canaria, 1987), 54-60; Revista Nacional de Arquitectura 10-11 extra. (1941): 31. Cf. asimismo Fernando Betancor Pérez, «Las Palmas de Gran Canaria y sus cines olvidados: aproximación a la historia de la arquitectura cinematográfica a través de los proyectos que quedaron en el papel» en Francisco Morales Padrón (coord.), XIII Coloquio de Historia Canario-Americana; VIII Congreso Internacional de Historia de América (AEA) (Las Palmas de Gran Canaria: Cabildo de Gran Canaria, 2000): 3033-3054.

18. Cf. Pedro Rodríguez Gutiérrez, «D. Tomás Machado y Méndez Fernández de Lugo, Dr. Arquitecto» (Tesis Doctoral, Universidad de La Laguna, 1990). 19. Cf. «Concurso de ideas y croquis para el campo de deportes del Real Madrid, F.C.- Acta de calificación y propuesta», Revista Nacional de Arquitectura 34 (1944): 360-361.

20. A la actuación de Perfumerías Gal, hay que sumar el edificio para la Coca-Cola en Madrid (1976); cf. VV.AA., La Arquitectura de la Industria, 19251965: registro DoCoMoMo Ibérico (Barcelona: Fundación Docomomo Ibérico, 2005), 205; VV.AA., Arquitectura de Madrid. Periferia, 323.

21. Destacan el parador de La Arruzafa en Córdoba (1960) y el de Albacete (1970); cf. José Ramón Moreno Pérez et al., 50 Años de arquitectura en Andalucía: 1936-1986 (Sevilla: Consejería de Obras Públicas y Transportes, 1987), 155; Manuel Sáinz de Vicuña y García Prieto, «Parador de Turismo en la ciudad de Albacete», Arquitectura 143 (1970): 20-22.

22. Mencionar el barrio del Niño Jesús en Madrid (1947/59) con Domínguez Salazar; la Plaza de Toros de Melilla (1947), con Federico Faci Iribarren, José Varela Feijoo, Alejandro Blond González y Jenaro Cristos de la Fuente, o la colonia Puerta Bonita en Madrid (1958), en colaboración con otros arquitectos como Coderch, Fisac o Sáenz de Oiza (cf. «Proyecto de urbanización. Barrio del Nińo Jesús (Madrid)», Revista Nacional de Arquitectura 69 (1947): 280-284; y «Proyecto de plaza de toros para la ciudad de Melilla,, Revista Nacional de Arquitectura $54-55$ (1946): 123-129; VV.AA., Arquitectura de Madrid. Periferia, 692). 23. Cf. «Gran San Blas. 7.484 viviendas. Madrid», Hogar y Arquitectura 22-23 (1959): sp.

24. Cf. VV.AA., Equipamientos I. Lugares públicos y nuevos programas: registro DOCOMOMO ibérico, 1925-1965 (Barcelona: Fundación Docomomo Ibérico, 2010), 146 .

25. Cf. Felipe García-Escudero Torroba y Ramón Vázquez Molezún, «Laboratorios I.T.T. de Standard Eléctrica», Arquitectura 172 (1973): 1-4. 


\section{Procedencia de las ilustraciones}

Fig. 01 y 07. Ángel de la Morena Suárez (h. 1949). Archivo familiar de Ángel de la Morena Suárez.

Fig. 02. Archivo del autor (2011).

Fig. 03. Elaboración del autor.

Fig. 04. Archivos familiares de los arquitectos.

Fig. 05. Archivo General del Ministerio de Fomento, El Rocío (Huelva). Sig. H-(9532)-RL.

Fig. 06 y 10. Archivo José Luis Torroba Álvarez.

Fig. 08. Archivo Municipal de Güímar (Tenerife). Sig. AMG 1943/8.

Fig. 09. Archivo General del Ministerio de Fomento, Zarza de Granadilla (Cáceres). Sig. CC-(9226)-RL.

Fig. 11. Archivo General del Ministerio de Fomento, Cardenete (Cuenca). Sig. CU-(9814)-RL.

Fig. 12. Archivo Manuel Sainz de Vicuña García-Prieto.

Fig. 13. Archivo General del Ministerio de Fomento, Cornago (Logroño). Sig. LO-(9886)-RL.

Fig. 14. Archivo General del Ministerio de Fomento, Llanes (Oviedo). Sig. O-(8407)-RL.

Fig. 15. Archivo General del Ministerio de Fomento, Celanova (Orense). Sig. OR-(9851)-RL.

\section{Sobre el autor}

Daniel Pinzón Ayala (1980, Ronda) es arquitecto (US, 2004), diplomado en Estudios Avanzados (US, 2009), Máster MAES (UCA, 2011) y miembro del Grupo de Investigación HUM-666: Ciudad, Arquitectura y Patrimonio Contemporáneos de la Universidad de Sevilla, para el que ha participado en diferentes proyectos de investigación (investigacioncontemporanea.com). Actualmente se encuentra desarrollando su tesis doctoral sobre las casas-cuartel de la Guardia Civil, inscrita en el Dpto. de Historia, Teoría y Composición Arquitectónica de dicha universidad y divulgada en diferentes congresos. Desarrolla su actividad profesional en Sevilla.

pinzonayala@hotmail.com 